\title{
Uniform estimate for characteristics-mixed finite element method for two-dimensional advection-dominated transport problems
}

Lei Gao ${ }^{1 *}$ and Fuji Xie ${ }^{2}$

\section{"Correspondence:}

sunnydoll@sjtu.edu.cn

'Shanghai Jiao Tong University, Dongchuan Road, Shanghai,

201100, People's Republic of China

Full list of author information is

available at the end of the article

\section{Springer}

\begin{abstract}
In this paper, we prove uniform optimal-order error estimates for characteristics-mixed finite element methods for two-dimensional convection-dominated diffusion equations. The generic constants in the error estimates do not explicitly depend on the scaling diffusion parameter $\varepsilon$, but depend linearly on certain Sobolev norms of the true solution. Combining the estimates with the stability estimates of the true solution, we prove that these constants depend only on the initial and the right-hand side data. Numerical experiments are presented to confirm our theoretical findings.
\end{abstract}

Keywords: uniform optimal-order error estimates; convection-dominated diffusion equations; characteristics-mixed finite element method; stability estimates; numerical experiments

\section{Introduction}

The characteristics-mixed finite element method is an efficient numerical scheme frequently used to solve the two-dimensional advection-dominated transport problem and tends to generate accurate numerical solutions for both the concentration and the flux if large time steps are used. Much research has been conducted on the error estimates of the characteristics-mixed finite element method in the context of linear convection-diffusion equations [1-3] or coupled systems for flow and transport in porous media [4-6]. Numerical examples suggest this kind of methods is effective and efficient. In particular, the characteristics-mixed finite element methods proposed in $[1,4,5]$ are mass-conservative, which is crucial in the practical applications. However, owing to the use of mixed elliptic projections whose estimates depend inversely on the diffusion coefficient $\varepsilon$, the corresponding error estimates derived for these methods should also depend on the inverse of $\varepsilon$ and could blow up when $\varepsilon$ approaches zero.

In order to overcome these difficulties, in this article, enlightened by the ideas in [7-10] for the Eulerian-Lagrangian localized adjoint method and the modified method of characteristics, we use an interpolation operator and Raviart-Thomas projection to replace the mixed elliptic projections and prove the uniform error estimates for characteristics-mixed finite element schemes for time dependent convection-diffusion equations with a periodic boundary condition. In these estimates, the generic constants depend linearly on certain

(C) The Author(s) 2017. This article is distributed under the terms of the Creative Commons Attribution 4.0 International License (http://creativecommons.org/licenses/by/4.0/), which permits unrestricted use, distribution, and reproduction in any medium, provided you give appropriate credit to the original author(s) and the source, provide a link to the Creative Commons license, and indicate if changes were made. 
Sobolev norms of the true solution but not explicitly on $\varepsilon$. Moreover, we take advantage of the stability estimates of the true solution and we prove the constants in these estimates depend only on certain Sobolev norms of the initial and the right-hand side data.

This article is organized as follows. In Section 2, we revisit the characteristics-mixed finite element scheme. In Section 3, we derive the uniform error estimates for the lowest Raviart-Thomas rectangle mixed finite element. In Section 4, combining the stability estimates of the true solution, we prove the constants depend only on the initial and the right-hand side data. In Section 5, we conduct the numerical experiments to verify our theoretical results. In Section 6, we summarize our main results and present the future work.

\section{Characteristics-mixed finite element scheme}

We consider the following two-dimensional convection-diffusion equation:

$$
\begin{aligned}
& \text { (a) } \frac{\partial c}{\partial t}+\mathbf{u}(\mathbf{x}, t) \cdot \nabla c-\varepsilon \nabla \cdot(D(\mathbf{x}, t) \nabla c)=f(\mathbf{x}, t), \quad(\mathbf{x}, t) \in \Omega \times(0, T] \\
& \text { (b) } c(\mathbf{x}, 0)=c_{0}(\mathbf{x}), \quad \mathbf{x} \in \Omega
\end{aligned}
$$

with a periodic boundary condition $[8,9,11]$. Here $\mathbf{x}=(x, y)$ and $\Omega=\left[a_{1}, a_{2}\right] \times\left[b_{1}, b_{2}\right]$ is a rectangular domain, $\partial \Omega$ represents the boundary of $\Omega \cdot \mathbf{u}(\mathbf{x}, t)=\left(u_{1}(\mathbf{x}, t), u_{2}(\mathbf{x}, t)\right)^{\prime}$ accounts for the velocity of the flow, where $(, \ldots,)^{\prime}$ denotes the transpose of a vector. The parameter $0<\varepsilon \ll 1$ characterizes the advection-dominance of problem (2.1). $f$ represents a source term, $c_{0}$ is the initial concentration and $c$ is the $\varepsilon$-dependent unknown function. $D$ denotes the diffusive coefficient tensor and satisfies $\alpha \chi^{\prime} \chi \leq \chi^{\prime} D \chi \leq \beta \chi^{\prime} \chi$ and $\alpha_{0} \chi^{\prime} \chi \leq \chi^{\prime} D^{-1} \chi \leq$ $\beta_{0} \chi^{\prime} \chi$ for any $\chi=\left(\chi_{1}, \chi_{2}\right)^{\prime} \in R^{2}$, where $\alpha, \alpha_{0}, \beta$ and $\beta_{0}>0$ are four positive constants.

Let $H^{s}(\Omega)$ denote the usual Sobolev space, provided the norm $\|\cdot\|_{s}$, for $s \geq 0$. When $s=0$, we use $\|\cdot\|$ to denote the corresponding $L^{2}$-norm. Furthermore, for any $\chi \in\left(L^{2}(\Omega)\right)^{2}$, we define the space $\mathbf{L}_{\varepsilon}^{2}(\Omega)=\left\{\chi: \varepsilon^{-\frac{1}{2}} \chi \in\left(L^{2}(\Omega)\right)^{2}\right\}$, with the norm $\|\chi\|_{\mathbf{L}_{\varepsilon}^{2}(\Omega)}=\varepsilon^{-\frac{1}{2}}\|\chi\|$. For any Banach space $X$, we introduce the following spaces that incorporate time dependence [12]:

$$
\begin{aligned}
& W_{p}^{k}\left(t_{1}, t_{2} ; X\right)=\left\{v:\left\|\frac{\partial^{l} v}{\partial t^{l}}(\cdot, t)\right\|_{X} \in L^{p}\left(t_{1}, t_{2}\right), 0 \leq l \leq k, 1 \leq p \leq \infty\right\}, \\
& \|v\|_{W_{p}^{k}\left(t_{1}, t_{2} ; X\right)}= \begin{cases}\sum_{l=0}^{k}\left(\int_{t_{1}}^{t_{2}}\left\|\frac{\partial^{l} v}{\partial t^{\prime}}(\cdot, t)\right\|_{X}^{p} d t\right)^{\frac{1}{p}}, & 1 \leq p<\infty, \\
\max _{0 \leq l \leq k} \operatorname{ess} \sup _{t \in\left(t_{1}, t_{2}\right)}\left\|\partial^{l^{l} v}(\cdot, t)\right\|_{X}, & p=\infty .\end{cases}
\end{aligned}
$$

To derive the characteristics-mixed variational formulation for problem (2.1), we let $\psi(\mathbf{x}, t)=\left(1+|\mathbf{u}(\mathbf{x}, t)|^{2}\right)^{\frac{1}{2}}=\left(1+u_{1}^{2}(\mathbf{x}, t)+u_{2}^{2}(\mathbf{x}, t)\right)^{\frac{1}{2}}$, and let the characteristic direction associated with the operator $c_{t}+\mathbf{u}(\mathbf{x}, t) \cdot \nabla c$ be denoted by $\tau(\mathbf{x}, t)$, so the total derivative along $\tau$ is

$$
\begin{aligned}
\frac{\partial}{\partial \tau(\mathbf{x}, t)} & =\frac{1}{\psi(\mathbf{x}, t)} \frac{\partial}{\partial t}+\frac{\mathbf{u}(\mathbf{x}, t)}{\psi(\mathbf{x}, t)} \cdot \nabla \\
& =\frac{1}{\psi(\mathbf{x}, t)} \frac{\partial}{\partial t}+\frac{u_{1}(\mathbf{x}, t)}{\psi(\mathbf{x}, t)} \frac{\partial}{\partial x}+\frac{u_{2}(\mathbf{x}, t)}{\psi(\mathbf{x}, t)} \frac{\partial}{\partial y},
\end{aligned}
$$

therefore (2.1a) can be written in the following form:

$$
\psi \frac{\partial c}{\partial \tau}-\varepsilon \nabla \cdot(D \nabla c)=f, \quad(\mathbf{x}, t) \in \Omega \times[0, T] .
$$


We define the space $\mathbf{H}=\left\{\chi: \chi \in\left(L^{2}(\Omega)\right)^{2}, \nabla \cdot \chi \in L^{2}(\Omega)\right\}$ and let $\mathbf{z}(\mathbf{x}, t)=-\varepsilon D(\mathbf{x}, t) \nabla c$. Then the mixed variational formulation corresponding to (2.1) is to find $(\mathbf{z}, c):(0, T] \rightarrow$ $\mathbf{H} \times L^{2}(\Omega)$ such that
(a) $\left(\psi \frac{\partial c}{\partial \boldsymbol{\tau}}, v\right)+(\nabla \cdot \mathbf{z}, v)=(f, v), \quad v \in L^{2}(\Omega)$
(b) $\left(D^{-1} \mathbf{z}, \chi\right)-\varepsilon(c, \nabla \cdot \chi)=0, \quad \chi \in \mathbf{H}$,
(c) $c(\mathbf{x}, 0)=c_{0}(\mathbf{x}), \quad \mathbf{x} \in \Omega$.

Take notice of the positive definiteness of $D^{-1}$. We use the Brezzi theorem to obtain the existence and uniqueness of solutions of system (2.2).

Now we define a uniform rectangular partition of $\Omega$ denoted by $\mathcal{T}_{h}: x_{i}=a_{1}+i \Delta x$, $i=0,1, \ldots, I, y_{j}=b_{1}+j \Delta y, j=0,1, \ldots, J$. Here $\Delta x=\left(a_{2}-a_{1}\right) / I, \Delta y=\left(b_{2}-b_{1}\right) / J$. For any $\Omega_{i j} \in \mathcal{T}_{h}$, let $\Omega_{i j}=\left(x_{i-1}, x_{i}\right) \times\left(y_{j-1}, y_{j}\right)$ and $h=\sqrt{(\Delta x)^{2}+(\Delta y)^{2}}, x_{i-\frac{1}{2}}=\left(x_{i-1}+x_{i}\right) / 2$, $y_{j-\frac{1}{2}}=\left(y_{j-1}+y_{j}\right) / 2$.

In this paper, we use the following lowest Raviart-Thomas rectangle mixed finite element spaces [13]:

$$
\begin{aligned}
& \mathbf{H}_{h}=\left\{\chi=\left(\chi_{1}, \chi_{2}\right)^{\prime} \in \mathbf{H}:\left.\chi_{1}\right|_{\Omega_{i j}}=c_{i j}^{1}+c_{i j}^{2} x \text { and }\left.\chi_{2}\right|_{\Omega_{i j}}=c_{i j}^{3}+c_{i j}^{4} y, \Omega_{i j} \in \mathcal{T}_{h}\right\}, \\
& V_{h}=\left\{v \in L^{2}(\Omega):\left.v\right|_{\Omega_{i j}}=c_{i j}^{0} \in \mathcal{Q}_{0}\left(\Omega_{i j}\right), \Omega_{i j} \in \mathcal{T}_{h}\right\},
\end{aligned}
$$

where $\mathcal{Q}_{k}\left(\Omega_{i j}\right)(k \geq 0)$ denotes the space of polynomials of a degree less than or equal to $k$ in each space direction and $c_{i j}^{l}(l=0,1,2,3,4)$ are constants.

To define the discrete scheme for problem (2.2), firstly, we use a uniform partition to divide the interval $[0, T]: t^{n}=n \Delta t, 0 \leq n \leq N$, where $\Delta t=T / N$. Based on this partition, we define a discrete norm as follows:

$$
\|\chi \chi\|_{L^{2}\left(0, T ; \mathbf{L}_{\varepsilon}^{2}(\Omega)\right)}=\left(\sum_{n=1}^{N} \Delta t\left\|\chi\left(t^{n}\right)\right\|_{\mathbf{L}_{\varepsilon}^{2}}^{2}\right)^{\frac{1}{2}} .
$$

Next, we consider a time step $\Delta t>0$ and approximate the solution at times $t^{n}=n \Delta t$. The characteristics derivative is approximated by a backward difference quotient along the approximate characteristic $\tau$ in the time stepping procedure [11].

Let

$$
\begin{aligned}
\overline{\mathbf{x}} & =(\bar{x}, \bar{y})=\mathbf{x}-\mathbf{u}\left(\mathbf{x}, t^{n}\right) \Delta t \\
& =\left(x-u_{1}\left(\mathbf{x}, t^{n}\right) \Delta t, y-u_{2}\left(\mathbf{x}, t^{n}\right) \Delta t\right),
\end{aligned}
$$

and note that

$$
\begin{aligned}
\psi^{n} \frac{\partial c^{n}}{\partial \tau} & \approx \psi^{n} \frac{c\left(\mathbf{x}, t^{n}\right)-c\left(\overline{\mathbf{x}}, t^{n-1}\right)}{\sqrt{|(\mathbf{x}-\overline{\mathbf{x}})|^{2}+\left(t^{n}-t^{n-1}\right)^{2}}} \\
& =\frac{c\left(\mathbf{x}, t^{n}\right)-c\left(\overline{\mathbf{x}}, t^{n-1}\right)}{\Delta t} .
\end{aligned}
$$

For simplicity, we use $c^{n}$ and $\bar{c}^{n-1}$ to replace $c\left(\mathbf{x}, t^{n}\right)$ and $c\left(\overline{\mathbf{x}}, t^{n-1}\right)$, respectively. 
So the characteristics-mixed finite element scheme is the determination of the map $\left(\mathbf{z}_{h}, c_{h}\right):\left\{t^{0}, t^{1}, \ldots, t^{N}\right\} \rightarrow \mathbf{H}_{h} \times V_{h}$ satisfying
(a) $\left(\frac{c_{h}^{n}-\bar{c}_{h}^{n-1}}{\Delta t}, v_{h}\right)+\left(\nabla \cdot \mathbf{z}_{h}^{n}, v_{h}\right)=\left(f^{n}, v_{h}\right), \quad v_{h} \in V_{h}, 1 \leq n \leq N$,
(b) $\left(D_{n}^{-1} \mathbf{z}_{h}^{n}, \chi_{h}\right)-\varepsilon\left(c_{h}^{n}, \nabla \cdot \chi_{h}\right)=0, \quad \chi_{h} \in \mathbf{H}_{h}, 0 \leq n \leq N$,
(c) $\quad c_{h}(\mathbf{x}, 0)=c_{0 h}(\mathbf{x}), \quad \mathbf{x} \in \Omega$.

The following theorem gives the existence and uniqueness of the solution of the discrete scheme (2.4).

Theorem 2.1 (see Theorem 2.1 of [2]) If the coefficient $D(\mathbf{x}, t)$ is uniformly positive definite, then there exists a unique solution of (2.4).

\section{Uniform error estimates for the discrete scheme}

In this section, we derive the uniform error estimates for the lowest Raviart-Thomas rectangle mixed finite element for problem (2.1) enclosed with a periodic boundary condition. In order to achieve this goal, we introduce the following projections at first:

We define $R_{h} v \in \mathcal{Q}_{0}\left(\Omega_{i j}\right)$ to be the piecewise constant interpolation for any $v \in C\left(\bar{\Omega}_{i j}\right)$, i.e.

$$
R_{h} v(x, y)=v\left(x_{i-\frac{1}{2}}, y_{j-\frac{1}{2}}\right)
$$

Let $\Pi_{h}$ be the Raviart-Thomas projection (see [13]) satisfying

$$
\left(\nabla \cdot\left(\chi-\Pi_{h} \chi\right), v_{h}\right)=0, \quad v_{h} \in V_{h}
$$

and

$$
\left\|\chi-\Pi_{h} \chi\right\| \leq Q h\|\chi\|_{1} .
$$

Let $\boldsymbol{\eta}=\mathbf{z}-\Pi_{h} \mathbf{z}, \xi_{h}=\Pi_{h} \mathbf{z}-\mathbf{z}_{h}, \rho=c-R_{h} c$ and $e_{h}=R_{h} c-c_{h}$. Then the following estimates are well known for $k=0,1$ (see $[14,15]$ ):

$$
\begin{aligned}
& \|\rho\|_{L^{\infty}\left(H^{k}(\Omega)\right)} \leq Q h^{1-k}\|c\|_{L^{\infty}\left(H^{1}(\Omega)\right)}, \\
& \left\|v_{h}\right\|_{1} \leq Q h^{-1}\left\|v_{h}\right\|, \quad v_{h} \in \mathcal{P}_{1},
\end{aligned}
$$

where all constants $Q$ are independent of $h$ and $\varepsilon$. Moreover, we denote by $\mathcal{P}_{k}$ the space of polynomials of a degree less than or equal to $k(k \geq 0)$.

Combining (3.3) with the assumption $\mathbf{z}=-\varepsilon \nabla c$, we have

$$
\|\boldsymbol{\eta}\|_{L^{\infty}\left(L^{2}(\Omega)\right)} \leq \varepsilon Q h\|c\|_{L^{\infty}\left(H^{2}(\Omega)\right)}
$$

With the help of these preliminary results we are in a position to prove the uniform error estimates for $\left\|c-c_{h}\right\|_{L^{\infty}\left(L^{2}\right)}$ and $\left\|\mathbf{z}-\mathbf{z}_{h}\right\|_{L^{2}\left(\mathbf{L}_{\varepsilon}^{2}\right)}$. By (2.2), (2.4) and (3.2), we obtain the error 
equations in the following form:

(a) $\left(D_{n}^{-1} \boldsymbol{\xi}_{h}^{n}, \boldsymbol{\chi}_{h}\right)-\varepsilon\left(e_{h}^{n}, \nabla \cdot \boldsymbol{\chi}_{h}\right)=\varepsilon\left(\rho^{n}, \nabla \cdot \chi_{h}\right)-\left(D_{n}^{-1} \eta^{n}, \chi_{h}\right), \quad \chi_{h} \in \mathbf{H}_{h}$,

(b) $\left(\frac{e_{h}^{n}-\bar{e}_{h}^{n-1}}{\Delta t}, v_{h}\right)+\left(\nabla \cdot \xi_{h}^{n}, v_{h}\right)$

$$
=\left(\frac{c^{n}-\bar{c}^{n-1}}{\Delta t}-\psi^{n} \frac{\partial c^{n}}{\partial \tau}, v_{h}\right)-\left(\frac{\rho^{n}-\bar{\rho}^{n-1}}{\Delta t}, v_{h}\right), \quad v_{h} \in V_{h} .
$$

We choose $\chi_{h}=\xi_{h}^{n}$ and $v_{h}=e_{h}^{n}$ in (3.7a) and (3.7b), respectively. We multiply (3.7b) by $\varepsilon$ and add (3.7a) and (3.7b) together to yield

$$
\begin{aligned}
\left(D_{n}^{-1} \xi_{h}^{n}, \xi_{h}^{n}\right)+\varepsilon\left(e_{h}^{n}, \frac{e_{h}^{n}-\bar{e}_{h}^{n-1}}{\Delta t}\right) \\
=\varepsilon\left(e_{h}^{n}, \frac{c^{n}-\bar{c}^{n-1}}{\Delta t}-\psi^{n} \frac{\partial c^{n}}{\partial \tau}\right)-\varepsilon\left(e_{h}^{n}, \frac{\rho^{n}-\bar{\rho}^{n-1}}{\Delta t}\right) \\
\quad+\varepsilon\left(\rho^{n}, \nabla \cdot \xi_{h}^{n}\right)-\left(D_{n}^{-1} \eta^{n}, \xi_{h}^{n}\right) .
\end{aligned}
$$

By the results of [2], we get

$$
\left\|\bar{e}_{h}^{n-1}\right\|^{2} \leq(1+Q \Delta t)\left\|e_{h}^{n-1}\right\|^{2} .
$$

Combining (3.9), the left-hand side of (3.8) is bounded by

$$
\begin{aligned}
& \left(D_{h}^{-1} \xi_{h}^{n}, \xi_{h}^{n}\right)+\varepsilon\left(e_{h}^{n}, \frac{e_{h}^{n}-\bar{e}_{h}^{n-1}}{\Delta t}\right) \\
& \quad \geq \frac{\varepsilon}{2 \Delta t}\left(\left(e_{h}^{n}, e_{h}^{n}\right)-\left(\bar{e}_{h}^{n-1}, \bar{e}_{h}^{n-1}\right)\right)+\alpha_{0}\left\|\xi_{h}^{n}\right\|^{2} \\
& \quad \geq \frac{\varepsilon}{2 \Delta t}\left(\left\|e_{h}^{n}\right\|^{2}-(1+Q \Delta t)\left\|e_{h}^{n-1}\right\|^{2}\right)+\alpha_{0}\left\|\xi_{h}^{n}\right\|^{2},
\end{aligned}
$$

where we use the following inequality in the first step:

$$
\frac{1}{2}\left(x^{2}-y^{2}\right) \leq \frac{1}{2}\left(x^{2}-y^{2}\right)+\frac{1}{2}(x-y)^{2}=(x-y) x, \quad \text { for any } x, y \in R .
$$

We plug (3.10) into (3.8) and use triangle inequalities to obtain

$$
\begin{aligned}
& \frac{\varepsilon}{2 \Delta t}\left(\left\|e_{h}^{n}\right\|^{2}-(1+Q \Delta t)\left\|e_{h}^{n-1}\right\|^{2}\right)+\alpha_{0}\left\|\xi_{h}^{n}\right\|^{2} \\
& \leq\left|\varepsilon\left(e_{h}^{n}, \frac{c^{n}-\bar{c}^{n-1}}{\Delta t}-\psi^{n} \frac{\partial c^{n}}{\partial \tau}\right)\right|+\left|\varepsilon\left(\rho^{n}, \nabla \cdot \xi_{h}^{n}\right)\right| \\
& \quad+\left|\varepsilon\left(e_{h}^{n}, \frac{\rho^{n}-\bar{\rho}^{n-1}}{\Delta t}\right)\right|+\left|\left(D_{n}^{-1} \eta^{n}, \xi_{h}^{n}\right)\right| .
\end{aligned}
$$


Next we begin to estimate the right-hand side of (3.11) term by term. The first and fourth terms in (3.11) are bounded directly by Hölder's inequality and the results derived in [11]:

$$
\begin{aligned}
& \left|\varepsilon\left(e_{h}^{n}, \frac{c^{n}-\bar{c}^{n-1}}{\Delta t}-\psi^{n} \frac{\partial c^{n}}{\partial \tau}\right)\right| \\
& \quad \leq \varepsilon Q\left\|\frac{\partial^{2} c}{\partial \tau^{2}}\right\|_{L^{2}\left(\Omega \times\left[t^{n-1}, t^{n}\right]\right)}^{2} \Delta t+\frac{\varepsilon}{2}\left\|e_{h}^{n}\right\|^{2}, \\
& \left|\left(D_{n}^{-1} \boldsymbol{\eta}^{n}, \boldsymbol{\xi}_{h}^{n}\right)\right| \leq Q\left\|\boldsymbol{\eta}^{n}\right\|^{2}+\frac{\alpha_{0}}{4}\left\|\boldsymbol{\xi}_{h}^{n}\right\|^{2} .
\end{aligned}
$$

Due to the discontinuity of $e_{h}^{n}$ and the influence of $\varepsilon$ in the third term on the right-hand side of (3.11), we are unable to employ either the $H^{-1}$-duality in [11] or the method in [2] to bound this term. Fortunately, Chen et al. proposed an effective method to overcome these difficulties. In Lemma 3.5 of [16], an $H^{1}$-function $\zeta_{h}^{n}$ was found to approximate $e_{h}^{n}$ and the error estimate of $\zeta_{h}^{n}-e_{h}^{n}$ in the $L^{2}(\Omega)$-norm was derived. We now introduce this result by the following lemma.

Lemma 3.1 (see [16]) If $e_{h}^{n}$ satisfies

$$
\left(D_{n}^{-1} \boldsymbol{\xi}_{h}^{n}, \boldsymbol{\chi}_{h}\right)-\varepsilon\left(e_{h}^{n}, \nabla \cdot \chi_{h}\right)=\varepsilon\left(\rho^{n}, \nabla \cdot \chi_{h}\right)-\left(D_{n}^{-1} \eta^{n}, \chi_{h}\right)
$$

then there exists a function $\zeta_{h}^{n} \in H^{1}(\Omega)$ and some constant $Q$ independent of $h, n$ and $\varepsilon$, such that

$$
\begin{aligned}
& \left\|\zeta_{h}^{n}\right\|_{1} \leq Q\left\|e_{h}^{n}\right\|, \\
& \left\|\zeta_{h}^{n}-e_{h}^{n}\right\| \leq Q h\left(\left\|e_{h}^{n}\right\|+h\|c\|_{L^{\infty}\left(H^{2}\right)}\right) .
\end{aligned}
$$

Based on Lemma 3.1, we set out to estimate the third term. We rewrite $\rho^{n}-\bar{\rho}^{n-1}$ as the $\operatorname{sum}\left(\rho^{n}-\rho^{n-1}\right)+\left(\rho^{n-1}-\bar{\rho}^{n-1}\right)$ and apply the triangle inequalities to get

$$
\begin{aligned}
\varepsilon\left|\left(e_{h}^{n}, \frac{\rho^{n}-\bar{\rho}^{n-1}}{\Delta t}\right)\right| \leq & \varepsilon\left|\left(\frac{\rho^{n}-\rho^{n-1}}{\Delta t}, e_{h}^{n}\right)\right|+\varepsilon\left|\left(\frac{\rho^{n-1}-\bar{\rho}^{n-1}}{\Delta t}, e_{h}^{n}\right)\right| \\
\leq & \frac{\varepsilon}{2}\left\|\frac{\rho^{n}-\rho^{n-1}}{\Delta t}\right\|^{2}+\frac{\varepsilon}{2}\left\|e_{h}^{n}\right\|^{2} \\
& +\varepsilon\left|\left(\frac{\rho^{n-1}-\bar{\rho}^{n-1}}{\Delta t}, \zeta_{h}^{n}\right)\right|+\varepsilon\left|\left(\frac{\rho^{n-1}-\bar{\rho}^{n-1}}{\Delta t}, \zeta_{h}^{n}-e_{h}^{n}\right)\right| \\
\leq & \frac{Q \varepsilon h^{2}}{\Delta t} \int_{t^{n-1}}^{t^{n}}\left\|c_{t}\right\|_{1}^{2} d t+Q \varepsilon\left\|e_{h}^{n}\right\|^{2} \\
& +\frac{Q}{\Delta t}\left\|\rho^{n-1}-\bar{\rho}^{n-1}\right\|{ }_{-1}\left\|e_{h}^{n}\right\| \\
& +\frac{Q h}{\Delta t}\left(\left\|e_{h}^{n}\right\|+h\|c\|_{L^{\infty}\left(H^{2}\right)}\right)\left\|\rho^{n-1}-\bar{\rho}^{n-1}\right\| .
\end{aligned}
$$

Following [2], we have

$$
\left\|\rho^{n-1}-\bar{\rho}^{n-1}\right\|_{-1} \leq Q\left\|\rho^{n-1}\right\| \Delta t \quad \text { and } \quad\left\|\rho^{n-1}-\bar{\rho}^{n-1}\right\| \leq Q\left\|\rho^{n-1}\right\| .
$$


We take these two estimates in (3.17) and suppose $h=O(\Delta t)$ to yield

$$
\begin{aligned}
\varepsilon\left|\left(e_{h}^{n}, \frac{\rho^{n}-\bar{\rho}^{n-1}}{\Delta t}\right)\right| \leq & Q\left\{\frac{\varepsilon h^{2}}{\Delta t} \int_{t^{n-1}}^{t^{n}}\left\|c_{t}\right\|_{1}^{2} d t+\varepsilon\left\|\rho^{n-1}\right\|\left\|e_{h}^{n}\right\|\right. \\
& \left.+\varepsilon \frac{h}{\Delta t}\left\|\rho^{n-1}\right\|\left(\left\|e_{h}^{n}\right\|+h\|c\|_{L^{\infty}\left(H^{2}\right)}\right)+\varepsilon\left\|e_{h}^{n}\right\|^{2}\right\} \\
\leq & Q\left\{\frac{\varepsilon h^{2}}{\Delta t} \int_{t^{n-1}}^{t^{n}}\left\|c_{t}\right\|_{1}^{2} d t+\varepsilon\left\|e_{h}^{n}\right\|^{2}\right. \\
& \left.+\varepsilon h\left\|e_{h}^{n}\right\|\|c\|_{L^{\infty}\left(H^{1}\right)}+\varepsilon h^{2}\|c\|_{L^{\infty}\left(H^{2}\right)}^{2}\right\} \\
\leq & Q \varepsilon h^{2}\left(\frac{1}{\Delta t} \int_{t^{n-1}}^{t^{n}}\left\|c_{t}\right\|_{1}^{2} d t+\|c\|_{L^{\infty}\left(H^{2}\right)}^{2}\right)+Q \varepsilon\left\|e_{h}^{n}\right\|^{2} .
\end{aligned}
$$

The second term in (3.11), resulting from the replacement of the mixed elliptic projection by the interpolation operator, cannot be estimated straightforwardly by Hölder's inequality, (3.4) or the inverse inequality (3.5). However, a straight estimate would bring about a suboptimal-order estimate owing to the appearance of $\nabla \cdot \boldsymbol{\xi}_{h}^{n}$. Hence, this term needs to be re-estimated by some new techniques.

In order to bound the second term, we use Taylor's expansion to prove Lemma 3.2, which plays an important role in raising the convergence rate.

Lemma 3.2 Let $R_{h}$ be the piecewise constant interpolation of $v \in H^{2}(\Omega)$. Then the following estimate holds:

$$
\left(v-R_{h} v, w\right) \leq Q h^{2}\|v\|_{2}\|w\|, \quad w \in V_{h} .
$$

Here $Q$ is a constant independent of $h$.

Proof We sum the left-hand side of (3.20) by parts

$$
\left(v-R_{h} v, w\right)=-\sum_{i, j} \int_{\Omega_{i j}}\left(R_{h}-I\right) v(x, y) w d x d y .
$$

By Taylor's expansion, we have the following expression for $v \in H^{2}\left(\Omega_{i j}\right)$ :

$$
\begin{aligned}
\left.\left(R_{h}-I\right) v(x, y)\right|_{\bar{\Omega}_{i j}}=\int_{x}^{x_{i-\frac{1}{2}}} & \frac{\partial v}{\partial \gamma_{1}}\left(\gamma_{1}, y\right) d \gamma_{1}+\int_{y}^{y_{j-\frac{1}{2}}} \frac{\partial v}{\partial \gamma_{2}}\left(x, \gamma_{2}\right) d \gamma_{2} \\
& +\int_{x}^{x_{i-\frac{1}{2}}} \int_{y}^{y_{j-\frac{1}{2}}} \frac{\partial^{2} v}{\partial \gamma_{1} \partial \gamma_{2}}\left(\gamma_{1}, \gamma_{2}\right) d \gamma_{2} d \gamma_{1} .
\end{aligned}
$$

We use (3.22) to replace $\left(R_{h}-I\right) v(x, y)$ in (3.21), and we can get

$$
\begin{aligned}
& \int_{\Omega_{i j}}\left(R_{h}-I\right) v(x, y) w d x d y \\
& \quad=\int_{\Omega_{i j}} w \int_{x}^{x}{ }^{i-\frac{1}{2}} \frac{\partial v}{\partial \gamma_{1}}\left(\gamma_{1}, y\right) d \gamma_{1} d x d y
\end{aligned}
$$




$$
\begin{aligned}
& +\int_{\Omega_{i j}} w \int_{y}^{y_{j-\frac{1}{2}}} \frac{\partial v}{\partial \gamma_{2}}\left(x, \gamma_{2}\right) d \gamma_{1} d x d y \\
& +\int_{\Omega_{i j}} w \int_{x}^{x_{i-\frac{1}{2}}} \int_{y}^{y_{j-\frac{1}{2}}} \frac{\partial^{2} v}{\partial \gamma_{1} \partial \gamma_{2}}\left(\gamma_{1}, \gamma_{2}\right) d \gamma_{2} d \gamma_{1} d x d y
\end{aligned}
$$

We estimate the third term at first, and use Hölder's inequality to obtain

$$
\int_{\Omega_{i j}} w \int_{x}^{x_{i-\frac{1}{2}}} \int_{y}^{y_{j-\frac{1}{2}}} \frac{\partial^{2} v}{\partial \gamma_{1} \partial \gamma_{2}}\left(\gamma_{1}, \gamma_{2}\right) d \gamma_{2} d \gamma_{1} d x d y \leq Q h^{2}\|w\|_{\Omega_{i j}}\|v\|_{2, \Omega_{i j}} .
$$

We need only to bound the first term on the right-hand side, since the second one can be bounded by symmetry. Note that $\int_{x_{i-1}}^{x_{i}}\left(x-x_{i-\frac{1}{2}}\right) d x=0$. We have

$$
\begin{aligned}
& \left|\int_{\Omega_{i j}} w \int_{x}^{x_{i-\frac{1}{2}}} \frac{\partial v}{\partial \gamma_{1}}\left(\gamma_{1}, y\right) d \gamma_{1} d x d y\right| \\
& \quad=\left|\int_{\Omega_{i j}} w \int_{x}^{x_{i-\frac{1}{2}}} \int_{\gamma_{1}}^{x_{i-\frac{1}{2}}} v_{z z}(z, y) d z d \gamma_{1} d x d y\right| \\
& \quad \leq Q h^{2}\|w\|_{\Omega_{i j}}\|v\|_{2, \Omega_{i j}} .
\end{aligned}
$$

Combining these estimates, we obtain the lemma.

Now we use Lemma 3.2 and the inverse inequality (3.5) to bound the second term on the right-hand side of (3.11) by

$$
\begin{aligned}
\left|\varepsilon\left(\rho^{n}, \nabla \cdot \xi_{h}^{n}\right)\right| \leq Q \varepsilon h^{2}\left\|c^{n}\right\|_{2}\left\|\nabla \cdot \xi_{h}^{n}\right\| \leq & Q \varepsilon h\|c\|_{L^{\infty}\left(H^{2}\right)}\left\|\xi_{h}^{n}\right\| \\
& \leq Q \varepsilon^{2} h^{2}\|c\|_{L^{\infty}\left(H^{2}\right)}^{2}+\frac{\alpha_{0}}{4}\left\|\xi_{h}^{n}\right\|^{2} \\
& \leq Q \varepsilon h^{2}\|c\|_{L^{\infty}\left(H^{2}\right)}^{2}+\frac{\alpha_{0}}{4}\left\|\xi_{h}^{n}\right\|^{2},
\end{aligned}
$$

where the relationship $0<\varepsilon^{2}<\varepsilon \ll 1$ has been used.

Putting (3.12), (3.13), (3.19) and (3.26) into (3.11) and rearranging, we have

$$
\begin{aligned}
& \frac{\varepsilon}{2 \Delta t}\left(\left\|e_{h}^{n}\right\|^{2}-\left\|e_{h}^{n-1}\right\|^{2}\right)+\frac{\alpha_{0}}{2}\left\|\xi_{h}^{n}\right\|^{2} \\
& \leq Q\left\{\left\|\eta^{n}\right\|^{2}+\varepsilon h^{2}\|c\|_{L^{\infty}\left(H^{2}\right)}^{2}+\varepsilon \Delta t \int_{t^{n-1}}^{t^{n}}\left\|\frac{\partial^{2} c}{\partial \tau^{2}}\right\|^{2} d t\right. \\
& \left.\quad+\varepsilon h^{2}\left(\frac{1}{\Delta t} \int_{t^{n-1}}^{t^{n}}\left\|c_{t}\right\|_{1}^{2} d t+\|c\|_{L^{\infty}\left(H^{2}\right)}^{2}\right)\right\}+\varepsilon Q\left\|e_{h}^{n}\right\|^{2}+\varepsilon Q\left\|e_{h}^{n-1}\right\|^{2}
\end{aligned}
$$

Multiplying $\frac{2 \Delta t}{\varepsilon}$ with the above inequality, summing from $n=1$ to $n=N$ and selecting the initial as $c_{h}(0)=R_{h} c(0)$, we obtain

$$
\begin{aligned}
\left\|e_{h}^{N}\right\|^{2} & +\varepsilon^{-1} \alpha_{0} \Delta t \sum_{n=1}^{N}\left\|\xi_{h}^{n}\right\|^{2} \\
& +\varepsilon h^{2}\left(\int_{0}^{T}\left\|c_{t}\right\|_{1}^{2} d t+\|c\|_{L^{\infty}\left(H^{2}\right)}^{2}\right)+Q \varepsilon \Delta t \sum_{n=0}^{N}\left\|e_{h}^{n}\right\|^{2}
\end{aligned}
$$




$$
\begin{aligned}
\leq & Q\left\{\varepsilon^{-1} \Delta t \sum_{n=1}^{N}\left\|\eta^{n}\right\|^{2}+h^{2}\|c\|_{L^{\infty}\left(H^{2}\right)}^{2}+(\Delta t)^{2} \int_{0}^{T}\left\|\frac{\partial^{2} c}{\partial \tau^{2}}\right\|^{2} d t\right. \\
& \left.+h^{2}\left(\int_{0}^{T}\left\|c_{t}\right\|_{1}^{2} d t+\|c\|_{L^{\infty}\left(H^{2}\right)}^{2}\right)\right\}+Q \Delta t \sum_{n=0}^{N}\left\|e_{h}^{n}\right\|^{2} \\
\leq & Q\left\{h^{2}\left(\|c\|_{L^{\infty}\left(H^{2}\right)}^{2}+\left\|c_{t}\right\|_{L^{2}\left(H^{1}\right)}^{2}\right)+(\Delta t)^{2}\left\|\frac{\partial^{2} c}{\partial \tau^{2}}\right\|_{L^{2}\left(L^{2}\right)}^{2}\right\} .
\end{aligned}
$$

By the Gronwall lemma, for sufficiently small $\Delta t$, we have

$$
\begin{aligned}
& \left\|e_{h}\right\|_{L^{\infty}\left(L^{2}\right)}+\left\|\boldsymbol{\xi}_{h}\right\|_{L^{2}\left(\mathbf{L}_{\varepsilon}^{2}\right)} \\
& \quad \leq Q\left\{\Delta t\left\|\frac{\partial^{2} c}{\partial \tau^{2}}\right\|_{L^{2}\left(L^{2}\right)}+h\left(\|c\|_{L^{\infty}\left(H^{2}\right)}+\left\|c_{t}\right\|_{L^{2}\left(H^{1}\right)}\right)\right\} .
\end{aligned}
$$

Combining the estimates for $\rho$ and $\eta$ in (3.4) and (3.6) with (3.27), assuming $h=O(\Delta t)$ and applying triangle inequalities, we obtain the following theorem.

Theorem 3.3 Let $(\mathbf{z}, c)$ and $\left(\mathrm{z}_{h}, c_{h}\right)$ be the solutions of (2.2) and (2.4), respectively. Then the following $\varepsilon$-uniform estimate holds for $h=O(\Delta t)$ and $c \in L^{\infty}\left(0, T ; H^{2}(\Omega)\right) \cap$ $H^{2}\left(0, T ; H^{1}(\Omega)\right)$ :

$$
\begin{aligned}
& \left\|c-c_{h}\right\|_{L^{\infty}\left(L^{2}\right)}+\left\|\mathbf{z}-\mathbf{z}_{h}\right\|_{L^{2}\left(\mathbf{L}_{\varepsilon}^{2}\right)} \\
& \quad \leq Q\left\{\Delta t\left\|\frac{\partial^{2} c}{\partial \tau^{2}}\right\|_{L^{2}\left(L^{2}\right)}+h\left(\|c\|_{L^{\infty}\left(H^{2}\right)}+\left\|c_{t}\right\|_{L^{2}\left(H^{1}\right)}\right)\right\} .
\end{aligned}
$$

Here the constant $Q$ is independent of $\varepsilon, h$ and $\Delta t$.

\section{Error estimate based on the data}

We have proved the uniform optimal-order error estimates for the lowest Raivart-Thomas rectangle mixed finite element. In this section, we will restrict these estimates by the initial $c_{0}$ and the right-hand side data $f$.

Theorem 4.1 If $D \in\left(W^{1, \infty}\left(0, T ; W^{3, \infty}(\Omega)\right)\right)^{4}, \mathbf{u} \in\left(W^{1, \infty}\left(0, T ; W^{2, \infty}(\Omega)\right)\right)^{2}, f \in H^{1}(0, T$; $\left.H^{3}(\Omega)\right)$ and $c_{0} \in H^{3}(\Omega)$, then the following error estimate holds:

$$
\begin{aligned}
& \left\|c-c_{h}\right\|_{L^{\infty}\left(L^{2}\right)}+\left\|\mathbf{z}-\mathbf{z}_{h}\right\|_{L^{2}\left(\mathbf{L}_{\varepsilon}^{2}\right)} \\
& \quad \leq Q\left\{h\left(\left\|c_{0}\right\|_{2}+\|f\|_{L^{2}\left(H^{2}\right)}\right)+\Delta t\left(\left\|c_{0}\right\|_{3}+\|f\|_{H^{1}\left(H^{3}\right)}\right)\right\} .
\end{aligned}
$$

Here the constant $Q$ is independent of $\varepsilon, h$ and $\Delta t$.

Proof We need to restrict $\|c\|_{L^{\infty}\left(H^{2}\right)},\|c\|_{L^{2}\left(H^{1}\right)}$ and $\left\|\frac{\partial^{2} c}{\partial \tau^{2}}\right\|_{L^{2}\left(L^{2}\right)}$ by $c_{0}$ and $f$. Firstly, we bound $\|c\|_{L^{\infty}\left(H^{2}\right)}$. We integrate equation (2.1a) that is multiplied by $c$ on $\Omega$ and combine Green's formula with the periodic boundary condition to yield

$$
\frac{1}{2} \frac{d}{d t}\|c\|^{2}+\varepsilon(D \nabla c, \nabla c)=(f, c)+\frac{1}{2}\left(\nabla \cdot \mathbf{u}, c^{2}\right) .
$$


We note the boundedness of $D$, multiply (4.2) by 2 and integrate the resulting equation from 0 to $t$ to obtain

$$
\begin{aligned}
\|c\|^{2}+2 \alpha \varepsilon \int_{0}^{t}\|\nabla c(s)\|^{2} d s \leq & \left\|c_{0}\right\|^{2}+\int_{0}^{t}\|f(s)\|^{2} d s \\
& +\left(1+\|\mathbf{u}\|_{L^{\infty}\left(W^{1, \infty}\right)}\right) \int_{0}^{t}\|c(s)\|^{2} d s .
\end{aligned}
$$

By the Gronwall lemma, we have

$$
\|c\|_{L^{\infty}\left(L^{2}\right)}^{2}+\varepsilon\|\nabla c\|_{L^{2}\left(L^{2}\right)}^{2} \leq Q_{1}\left(\|c\|_{0}^{2}+\|f\|_{L^{2}\left(L^{2}\right)}^{2}\right)
$$

where $Q_{1}=\frac{1}{\{\max 1,2 \alpha\}}$.

We differentiate equation (2.1a) with respect to $x$ and $y$, respectively, and we have

$$
c_{t x}+\mathbf{u}_{x} \cdot \nabla c+\mathbf{u} \cdot \nabla c_{x}-\varepsilon \nabla \cdot\left(D_{x} \nabla c\right)-\varepsilon \nabla \cdot\left(D \nabla c_{x}\right)=f_{x}
$$

and

$$
c_{t y}+\mathbf{u}_{y} \cdot \nabla c+\mathbf{u} \cdot \nabla c_{y}-\varepsilon \nabla \cdot\left(D_{y} \nabla c\right)-\varepsilon \nabla \cdot\left(D \nabla c_{y}\right)=f_{y} .
$$

Multiplying (4.4) by $c_{x}$ and (4.6) by $c_{y}$, integrating the resulting equations over $\Omega$ and summing them, we derive

$$
\begin{aligned}
& \frac{1}{2} \frac{d}{d t}\|\nabla c\|^{2}+\varepsilon\left(D \nabla c_{x}, \nabla c_{x}\right)+\varepsilon\left(D \nabla c_{y}, \nabla c_{y}\right) \\
& \quad=(\nabla f, \nabla c)-(\nabla \mathbf{u} \nabla c, \nabla c)+\frac{1}{2}\left(\nabla \cdot \mathbf{u},(\nabla c)^{2}\right)-\varepsilon\left(D_{x} \nabla c, \nabla c_{x}\right)-\varepsilon\left(D_{y} \nabla c, \nabla c_{y}\right),
\end{aligned}
$$

where we use Green's formula and the periodic boundary condition again. By Cauchy's inequality, we have

$$
\begin{aligned}
\frac{1}{2} \frac{d}{d t}\|\nabla c\|^{2}+\alpha \varepsilon\left(\left\|\nabla c_{x}\right\|^{2}+\left\|\nabla c_{y}\right\|^{2}\right) \\
\leq \frac{1}{2}\|\nabla f\|^{2}+\left(\frac{1}{2}+\|\nabla \mathbf{u}\|_{L^{\infty}\left(L^{\infty}\right)}+\frac{1}{2}\|\nabla \cdot \mathbf{u}\|_{L^{\infty}\left(L^{\infty}\right)}\right. \\
\left.\quad+\frac{\left\|D_{x}\right\|_{L^{\infty}\left(L^{\infty}\right)+}+\left\|D_{y}\right\|_{L^{\infty}\left(L^{\infty}\right)}}{2 \alpha}\right)\|\nabla c\|^{2} \\
\leq \frac{1}{2}\|\nabla f\|^{2}+Q_{2}\|\nabla c\|^{2} .
\end{aligned}
$$

Here $Q_{2}=\frac{1}{2}+\frac{5}{2}\|\mathbf{u}\|_{L^{\infty}\left(W^{1, \infty}\right)}+\frac{1}{\alpha}\|D\|_{L^{\infty}\left(W^{1, \infty}\right)}^{2}$.

We multiply the above inequality by 2, integrate it from 0 to $t$ and use Gronwall's lemma to yield

$$
\|\nabla c\|_{L^{\infty}\left(L^{2}\right)}^{2}+\alpha \varepsilon\left(\left\|\nabla c_{x}\right\|_{L^{2}\left(L^{2}\right)}^{2}+\left\|\nabla c_{y}\right\|_{L^{2}\left(L^{2}\right)}^{2}\right) \leq\left\|c_{0}\right\|_{1}^{2}+\|f\|_{L^{2}\left(H^{1}\right)}^{2} .
$$


We use the same method to derive the following bounds:

$$
\begin{aligned}
& \alpha \varepsilon\left(\left\|\nabla c_{x x}\right\|_{L^{2}\left(L^{2}\right)}^{2}+2\left\|\nabla c_{x y}\right\|_{L^{2}\left(L^{2}\right)}^{2}+\left\|\nabla c_{y y}\right\|_{L^{2}\left(L^{2}\right)}^{2}\right) \\
& \quad+\|\nabla(\nabla c)\|_{L^{\infty}\left(L^{2}\right)}^{2} \leq Q_{3}\left(\left\|c_{0}\right\|_{2}^{2}+\|f\|_{L^{2}\left(H^{2}\right)}^{2}\right), \\
& \alpha \varepsilon\left(\left\|\nabla c_{x x x}\right\|_{L^{2}\left(L^{2}\right)}^{2}+\left\|\nabla c_{x x y}\right\|_{L^{2}\left(L^{2}\right)}^{2}+\left\|\nabla c_{x y y}\right\|_{L^{2}\left(L^{2}\right)}^{2}+\left\|\nabla c_{y y y}\right\|_{L^{2}\left(L^{2}\right)}^{2}\right) \\
& \quad+|c|_{L^{\infty}\left(H^{3}\right)}^{2} \leq Q_{3}\left(\left\|c_{0}\right\|_{3}^{2}+\|f\|_{L^{2}\left(H^{3}\right)}^{2}\right) .
\end{aligned}
$$

Here $Q_{3}=1+2\|\mathbf{u}\|_{L^{\infty}\left(W^{2, \infty}\right)}+\frac{5\|D\|_{L^{\infty}\left(W^{2, \infty}\right)}^{2}}{2 \alpha}$ and $Q_{4}=1+\left(4+2 Q_{3}\right)\left(\frac{9}{2}\|\mathbf{u}\|_{L^{\infty}\left(W^{3, \infty}\right)}+\right.$ $\left.\frac{23}{\alpha}\|D\|_{L^{\infty}\left(W^{3, \infty}\right)}^{2}\right)$.

By the estimates (4.4), (4.9), (4.10) and the definitions of the $H^{2}$-norm and the $H^{3}$-norm, we derive the two inequalities

$$
\begin{aligned}
\|c\|_{L^{\infty}\left(H^{2}\right)}^{2} & =\|c\|_{L^{\infty}\left(L^{2}\right)}^{2}+\|\nabla c\|_{L^{\infty}\left(L^{2}\right)}^{2}+\|\nabla(\nabla c)\|_{L^{\infty}\left(L^{2}\right)}^{2} \\
& \leq\left(1+Q_{1}+Q_{3}\right)\left(\left\|c_{0}\right\|_{2}^{2}+\|f\|_{L^{2}\left(H^{2}\right)}^{2}\right)
\end{aligned}
$$

and

$$
\begin{aligned}
\|c\|_{L^{\infty}\left(H^{3}\right)}^{2} & =\|c\|_{L^{\infty}\left(H^{2}\right)}^{2}+|c|_{L^{\infty}\left(H^{3}\right)}^{2} \\
& \leq\left(1+Q_{1}+Q_{3}+Q_{4}\right)\left(\left\|c_{0}\right\|_{3}^{2}+\|f\|_{L^{2}\left(H^{3}\right)}^{2}\right) .
\end{aligned}
$$

Therefore, we have

$$
\|c\|_{L^{\infty}\left(H^{2}\right)} \leq Q_{5}\left(\left\|c_{0}\right\|_{2}+\|f\|_{L^{2}\left(H^{2}\right)}\right)
$$

and

$$
\|c\|_{L^{\infty}\left(H^{3}\right)} \leq Q_{6}\left(\left\|c_{0}\right\|_{3}+\|f\|_{L^{2}\left(H^{3}\right)}\right)
$$

where $Q_{5}=\sqrt{1+Q_{1}+Q_{3}}$ and $Q_{6}=\sqrt{1+Q_{1}+Q_{3}+Q_{4}}$.

In order to bound $\left\|c_{t}\right\|_{L^{2}\left(H^{1}\right)}$, we use equation (2.1a) to express $c_{t}$ and apply the estimates (4.10) and (4.14) to gain

$$
\begin{aligned}
\left\|c_{t}\right\|_{L^{2}\left(H^{1}\right)}= & \|f-\mathbf{u} \cdot \nabla c+\varepsilon \nabla \cdot(D \nabla c)\|_{L^{2}\left(H^{1}\right)} \\
\leq & \|f\|_{L^{2}\left(H^{1}\right)}+\|\mathbf{u}\|_{L^{\infty}\left(W^{1, \infty}\right)}\|\nabla c\|_{L^{2}\left(H^{1}\right)}+\varepsilon\|D\|_{L^{\infty}\left(W^{2, \infty}\right)}\|\nabla c\|_{L^{2}\left(H^{1}\right)} \\
& +\varepsilon\|D\|_{L^{\infty}\left(W^{1, \infty}\right)}\left(\left\|c_{x x}\right\|_{L^{2}\left(H^{1}\right)}+2\left\|c_{x y}\right\|_{L^{2}\left(H^{1}\right)}+\left\|c_{y y}\right\|_{L^{2}\left(H^{1}\right)}\right) \\
\leq & \|f\|_{L^{2}\left(H^{1}\right)}+\left(\|\mathbf{u}\|_{L^{\infty}\left(W^{1, \infty}\right)}+5\|D\|_{L^{\infty}\left(W^{2, \infty}\right)}\right)\|c\|_{L^{\infty}\left(H^{2}\right)} \\
& +\frac{4 Q_{3}}{\alpha}\|D\|_{L^{\infty}\left(W^{1, \infty}\right)}\left(\left\|c_{0}\right\|_{2}+\|f\|_{L^{2}\left(H^{2}\right)}\right) \\
\leq & Q_{7}\left(\left\|c_{0}\right\|_{2}+\|f\|_{L^{2}\left(H^{2}\right)}\right),
\end{aligned}
$$

where $Q_{7}=1+Q_{5}\|\mathbf{u}\|_{L^{\infty}\left(W^{1, \infty}\right)}+\left(5 Q_{5}+\frac{4 Q_{3}}{\alpha}\right)\|D\|_{L^{\infty}\left(W^{2, \infty}\right)}$. Similarly, we have

$$
\left\|c_{t}\right\|_{L^{2}\left(H^{2}\right)} \leq Q_{8}\left(\left\|c_{0}\right\|_{3}+\|f\|_{L^{2}\left(H^{3}\right)}\right) .
$$


Here $Q_{8}=1+\left(Q_{6}+\frac{4(1+\alpha)}{\alpha} \sqrt{Q_{3}}+\frac{8 \sqrt{Q_{4}}}{\alpha}\right)\|D\|_{L^{\infty}\left(W^{3, \infty}\right)}+Q_{6}\|\mathbf{u}\|_{L^{\infty}\left(W^{2, \infty}\right)}$.

To prove the bound of $\left\|\frac{\partial^{2} c}{\partial \tau^{2}}\right\|_{L^{2}\left(L^{2}\right)}$, we differentiate the governing equation in (2.1) with respect to $t$ and express $c_{t t}$ in terms of the spatial derivatives and combine (4.16) with (4.17) to obtain

$$
\begin{aligned}
\left\|c_{t t}\right\|_{L^{2}\left(L^{2}\right)} & =\left\|f_{t}-(\mathbf{u} \nabla c)_{t}-\varepsilon(\nabla \cdot(D \nabla c))_{t}\right\|_{L^{2}\left(L^{2}\right)} \\
& \leq\left\|f_{t}\right\|_{L^{2}\left(L^{2}\right)}+2\|\mathbf{u}\|_{W^{1, \infty}\left(L^{\infty}\right)}\|c\|_{H^{1}\left(H^{1}\right)}+4\|D\|_{W^{1, \infty}\left(W^{1, \infty}\right)}\|c\|_{H^{1}\left(H^{2}\right)} \\
& \leq Q_{9}\left(\left\|c_{0}\right\|_{3}+\|f\|_{H^{1}\left(H^{3}\right)}\right) .
\end{aligned}
$$

Here $Q_{9}=1+2 Q_{7}\|\mathbf{u}\|_{W^{1, \infty}\left(L^{\infty}\right)}+4 Q_{8}\|D\|_{W^{1, \infty}\left(W^{1, \infty}\right)}$.

Note that $\left\|\frac{\partial^{2} c}{\partial \tau^{2}}\right\|_{L^{2}\left(L^{2}\right)} \leq Q\left\|c_{t t}\right\|_{L^{2}\left(L^{2}\right)}$, so combining (4.14) with (4.16) and (4.18), we obtain (4.1).

\section{Numerical experiments}

In this section, we carry out numerical experiments to verify our theoretical results. The initial data are chosen as

$$
\Omega=[-0.5,0.5] \times[-0.5,0.5], \quad T=\frac{\pi}{2}, \quad \mathbf{u}=(-4 y, 4 x)^{\prime}, \quad D=1,
$$

and the exact solution is given by

$$
\begin{aligned}
c(x, y, t)= & \frac{0.004}{0.004+4 \varepsilon t} \\
& \times \exp \left\{-\frac{(x \cos (4 t)+y \sin (4 t)+0.25)^{2}+(-x \sin (4 t)+y \cos (4 t))^{2}}{0.004+4 \varepsilon t}\right\} .
\end{aligned}
$$

Let $t=0$. Then we get the initial value

$$
c_{0}(x, y)=\exp \left\{-\frac{(x+0.25)^{2}+y^{2}}{0.004}\right\}
$$

By inserting (5.1) into equation (2.1a), we derive the source term $f=0$.

In our numerical convergence analysis, we use the lowest Raviart-Thomas rectangle mixed finite element space to fit the convergence rate

$$
\begin{aligned}
& \left\|c(\mathbf{x}, T)-c_{h}(\mathbf{x}, T)\right\| \leq \bar{Q}_{1}(h+\Delta t), \\
& \|\| \mathbf{z}(\mathbf{x}, T)-\mathbf{z}_{h}(\mathbf{x}, T) \|_{\mathbf{L}_{\varepsilon}^{2}} \leq \bar{Q}_{2}(h+\Delta t) .
\end{aligned}
$$

Here we choose $h=\Delta t$ and $\varepsilon=0.001,0.0005,0.0001,0.00005$. The results are presented in Tables 1-2 which show that we nearly obtain the same convergence rates and constants for different $\varepsilon$. This suggests that our derived error estimates do not depend explicitly on $\varepsilon$ as predicted by Theorem 3.3. Furthermore, these results show that the characteristics-mixed finite element scheme possesses the first-order accuracy in space and time as predicted by the theorem in Section 3 . 
Table 1 The convergence rate for $\left\|c-c_{h}\right\|$ in the two-dimensional case

\begin{tabular}{lllll}
\hline $\boldsymbol{h =} \boldsymbol{\Delta} \boldsymbol{t}$ & $\left\|\boldsymbol{c}-\boldsymbol{c}_{\boldsymbol{h}}\right\|$ & & & \\
\cline { 2 - 5 } & $\boldsymbol{\varepsilon}=\mathbf{0 . 0 0 1}$ & $\boldsymbol{\varepsilon}=\mathbf{0 . 0 0 0 5}$ & $\boldsymbol{\varepsilon}=\mathbf{0 . 0 0 0 1}$ & $\boldsymbol{\varepsilon}=\mathbf{0 . 0 0 0 0 5}$ \\
\hline $1 / 16$ & 0.0025 & 0.0025 & 0.0026 & 0.0026 \\
$1 / 32$ & 0.0017 & 0.0017 & 0.0017 & 0.0017 \\
$1 / 48$ & $8.8193 \mathrm{e}-04$ & $8.6351 \mathrm{e}-04$ & $8.6170 \mathrm{e}-04$ & $8.6252 \mathrm{e}-04$ \\
$1 / 64$ & $6.7982 \mathrm{e}-04$ & $6.5229 \mathrm{e}-04$ & $6.4625 \mathrm{e}-04$ & $6.4692 \mathrm{e}-04$ \\
& & & Convergence rate 0.9512 & Constant $\bar{Q}_{1}=0.0205$ \\
\hline
\end{tabular}

Table 2 The convergence rate for $\left\|z-z_{h}\right\|_{L_{\varepsilon}^{2}}$ in the two-dimensional case

\begin{tabular}{lllll}
\hline $\boldsymbol{h =} \boldsymbol{\Delta} \boldsymbol{t}$ & $\| \mathbf{z}-\mathbf{z}_{\boldsymbol{h} \|_{\mathbf{L} \boldsymbol{\varepsilon}}}$ & & & \\
\cline { 2 - 5 } & $\boldsymbol{\varepsilon}=\mathbf{0 . 0 0 1}$ & $\boldsymbol{\varepsilon}=\mathbf{0 . 0 0 0 5}$ & $\boldsymbol{\varepsilon}=\mathbf{0 . 0 0 0 1}$ & $\boldsymbol{\varepsilon}=\mathbf{0 . 0 0 0 0 5}$ \\
\hline $1 / 16$ & $4.8981 \mathrm{e}-04$ & $5.0423 \mathrm{e}-04$ & $5.1575 \mathrm{e}-04$ & $5.1719 \mathrm{e}-04$ \\
$1 / 32$ & $3.1965 \mathrm{e}-04$ & $3.3192 \mathrm{e}-04$ & $3.4191 \mathrm{e}-04$ & $3.4317 \mathrm{e}-04$ \\
$1 / 48$ & $1.5467 \mathrm{e}-04$ & $1.6197 \mathrm{e}-04$ & $1.6828 \mathrm{e}-04$ & $1.6911 \mathrm{e}-04$ \\
$1 / 64$ & $1.1503 \mathrm{e}-04$ & $1.2055 \mathrm{e}-04$ & $1.2548 \mathrm{e}-04$ & $1.2614 \mathrm{e}-04$ \\
& & & Convergence rate 1.0526 & Constant $\bar{Q}_{2}=0.0041$ \\
\hline
\end{tabular}

\section{Concluding remarks}

In this paper, we use the lowest Raviart-Thomas rectangle mixed finite element to approximate problem (2.1) and derive the uniform error estimates for the characteristics-mixed finite element schemes. Furthermore, combining the stability estimates of the true solution, we prove the generic constants depend only on the initial and right-hand side data. In the end, we conduct numerical experiments to certify our theoretical results. However, we see that the analysis in this paper is carried out on a rectangular domain with a uniform rectangular partition, the extension to the irregular region with a triangulation is open for future work.

\section{Acknowledgements}

We wish to thank the editor and reviewers for their precious comments and suggestions. This work was supported by the National Natural Science Foundation of China under Grant 71373158.

\section{Competing interests}

The authors declare that they have no competing interests.

\section{Authors' contributions}

All authors read and approved the final manuscript.

\section{Author details}

${ }^{1}$ Shanghai Jiao Tong University, Dongchuan Road, Shanghai, 201100, People's Republic of China. ${ }^{2}$ Anti College of

Economics \& Mangagement, Shanghai Jiao Tong University, Huashan Road, Shanghai, 200030, People's Republic of China.

\section{Publisher's Note}

Springer Nature remains neutral with regard to jurisdictional claims in published maps and institutional affiliations.

Received: 7 March 2017 Accepted: 15 August 2017 Published online: 30 August 2017

\section{References}

1. Arbogast, T, Wheeler, MF: A characteristics-mixed finite element method for advection-dominated transport problems. SIAM J. Numer. Anal. 32, 404-424 (1995)

2. Chen, HZ, Zhou, ZJ, Wang, H, Man, HY: An optimal-order error estimate for a family of characteristic-mixed methods to transient convection-diffusion problem. Discrete Contin. Dyn. Syst., Ser. B 15, 325-341 (2011)

3. Yang, D: A characteristic mixed method with dynamic finite-element space for convection-dominated diffusion problems. J. Comput. Appl. Math. 43, 343-353 (1992)

4. Douglas, J Jr, Huang, C-S, Pereira, F: The modified method of characteristics with adjusted advection. Numer. Math. 83, 353-369 (1999) 
5. Douglas, J Jr, Furtado, F, Pereira, F: On the numerical simulation of waterflooding of heterogeneous petroleum reservoirs. Comput. Geosci. 1, 155-190 (1997)

6. Ewing, RE, Russell, TF, Wheeler, MF: Convergence analysis of an approximation of miscible displacement in porous media by mixed finite elements and a modified method of characteristics. Comput. Methods Appl. Mech. Eng. 47 73-92 (1984)

7. Wang, $\mathrm{H}$, Wang, K: Uniform estimates for Eulerian-Lagrangian methods for singularly perturbed time-dependent problems. SIAM J. Numer. Anal. 45, 1305-1329 (2007)

8. Wang, K, Wang, H: A uniform estimate for the ELLAM scheme for transport equations. Numer. Methods Partial Differ. Equ. 24, 535-554 (2008)

9. Wang, K, Wang, H: Uniform estimates for a family of Eulerian-Lagrangian methods for time-dependent convection-diffusion equations with degenerate diffusion. IMA J. Numer. Anal. 31, 1006-1037 (2011)

10. Wang, $\mathrm{K}$, Wang, $\mathrm{H}$ : A uniform estimate for the MMOC for two-dimensional advection-diffusion equations. Numer Methods Partial Differ. Equ. 26, 1054-1069 (2010)

11. Douglas, J Jr, Russell, TF: Numerical methods for convection-dominated diffusion problems based on combining the method of characteristics with finite element or finite difference procedures. SIAM J. Numer. Anal. 19, 871-885 (1982)

12. Evans, LC: Partial Differential Equations. Am. Math. Soc., Providence (1998)

13. Raviat, PA, Thomas, JM: A mixed finite element method for 2 nd order elliptic problems. In: Mathematical Aspects of Finite Element Methods. Lecture Notes in Mathematics, vol. 606, pp. 292-315 (1977)

14. Ciarlet, PG: The Finite Element Method for Elliptic Problems. North-Holland, Amsterdam (1978)

15. DeVore, RA, Lorentz, GG: Constructive Approximation. Springer, Berlin (1993)

16. Chen, $H Z$, Gao, L, Wang, H: Uniform estimates for characteristics-mixed finite method for transient advection-dominated diffusion problems in two-dimensional space. Appl. Math. Comput. 280, 86-102 (2016)

\section{Submit your manuscript to a SpringerOpen ${ }^{\circ}$ journal and benefit from:}

- Convenient online submission

- Rigorous peer review

- Open access: articles freely available online

- High visibility within the field

- Retaining the copyright to your article

Submit your next manuscript at $\gg$ springeropen.com 